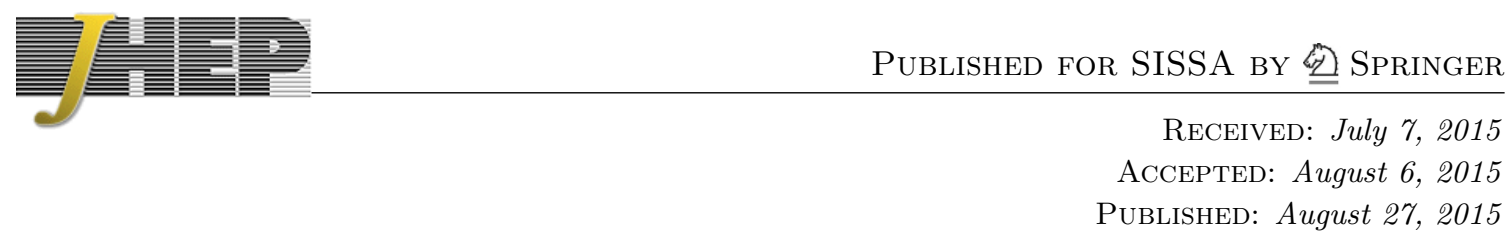

\title{
Challenges for the Nelson-Barr mechanism
}

\author{
Michael Dine ${ }^{a}$ and Patrick Draper ${ }^{a, b}$ \\ ${ }^{a}$ Santa Cruz Institute for Particle Physics and Department of Physics, University of California, \\ Santa Cruz, CA 93106, U.S.A. \\ ${ }^{b}$ Department of Physics, University of California, \\ Santa Barbara, CA 93106, U.S.A. \\ E-mail: mdine@ucsc.edu, pidraper@physics.ucsb.edu
}

ABSTRACT: The axion and $m_{u}=0$ solutions to the strong CP problem have been subject to the most careful scrutiny and critique. Basic theoretical issues include hierarchy and finetuning problems, quality and genericity of symmetries, and compatibility with solutions to the electroweak hierarchy problem. We study the similar set of challenges for solutions to strong CP based on spontaneous CP violation and the Nelson-Barr mechanism. Some of our observations have appeared in the literature previously, and others are new; our purpose is to collect and analyze the issues as a whole and provide an assessment of the most plausible settings for the Nelson-Barr solution.

Keywords: Beyond Standard Model, Quark Masses and SM Parameters, CP violation

ARXIV EPRINT: 1506.05433 


\section{Contents}

1 Introduction 1

2 The essence of the Nelson-Barr mechanism 3

3 Nonsupersymmetric Nelson-Barr models $\quad 5$

3.1 Basic challenges 5

3.2 Radiative corrections to $\theta$ in non-supersymmetric theories 6

$\begin{array}{lll}3.3 & \text { Models with strong dynamics } & 7\end{array}$

4 CP in supersymmetric theories: axions, moduli, and $\theta$ at tree level 9

5 SUSY Nelson-Barr models $\quad 11$

$\begin{array}{ll}5.1 \text { Breaking of CP and } Z_{N} \text { in SUSY } & 11\end{array}$

$\begin{array}{lll}\text { 5.1.1 CP broken by supersymmetry-conserving dynamics } & 12\end{array}$

$\begin{array}{ll}\text { 5.1.2 Theories with flat directions } & 12\end{array}$

$\begin{array}{lll}5.2 & \text { Breaking of supersymmetry } & 13\end{array}$

$\begin{array}{lll}5.3 & \text { Loop corrections in supersymmetric theories } & 14\end{array}$

$\begin{array}{llr}6 & \text { Conclusions } & 16\end{array}$

\section{Introduction}

There are various naturalness problems of the Standard Model (SM), including the cosmological constant problem, the hierarchy problem, the hierarchies in the quark and lepton mass matrices, and the strong CP problem. Of these, the last is special. Even modest changes in the cosmological constant would drastically alter the world around us. Similarly, the values of the weak scale and the light quark and lepton masses play critical roles in a range of phenomena. But if the $\mathrm{CP}$-violating parameter $\bar{\theta}$ were, say, $10^{-3}$, there would be no appreciable change in nuclear physics.

Theorists may put forward complicated explanations for the smallness of $\bar{\theta}$, with many additional degrees of freedom, complicated symmetries, and some amount of fine tuning, but this activity is not particularly satisfying. More compelling would be a theory in which the the smallness of $\bar{\theta}$ emerged as an accidental consequence of other structure in a physical theory: an explanation of flavor or dark matter, for example. We will refer to this (presently hypothetical) phenomenon as incidental CP conservation.

Most attention has focussed on three solutions to the strong CP problem: the possibility of a massless up quark, the Peccei-Quinn (PQ) solution with its associated axion [1, 2], and spontaneous $\mathrm{CP}$ or $\mathrm{P}$ violation with a protection mechanism for $\bar{\theta}$ [3-13]. The first two solutions require that the theory possess an approximate $\mathrm{U}(1)$ symmetry, the violation 
of which is primarily due to the QCD anomaly. If the symmetry is not spontaneously broken at scales above the QCD scale, there must be one or more very light quarks. This is usually stated as the requirement that the $u$ quark mass vanishes, but the more precise statement is that at scales beyond a few $\mathrm{GeV}, \frac{m_{u}}{m_{d}}<10^{-10}$. Apart from any theoretical issues, the possibility of a massless quark is strongly disfavored by lattice calculations [14]. If the chiral symmetry is nonlinearly realized, there is a light axion $[1,2]$. The potential for this axion determines $\bar{\theta}$.

The third proposed solution is that $\mathrm{CP}$ or $\mathrm{P}$ is spontaneously broken and $\bar{\theta}$ is protected by extra structure [3-7], the most common example of which is the Nelson-Barr (NB) mechanism [8-10] in the case of spontaneous CP violation. ${ }^{1}$ Since the underlying theory is CP-conserving, the "bare" $\bar{\theta}$ parameter vanishes. CP must then be spontaneously broken in a way that ensures a small effective $\bar{\theta}$ while allowing an order one phase in the CKM matrix (and a mechanism for baryogenesis) [3-10]. The NB proposal is striking in that it seeks to solve the strong CP problem with no low energy consequence, unlike the axion and $m_{u}=0$ solutions. On the other hand, in this paper, we will see some relations between these proposals. ${ }^{2}$

Setting aside the possibility that $m_{u}=0$ leaves the PQ and NB proposals. As currently implemented in an array of models, neither is completely satisfactory from a theoretical point of view; certainly neither is obviously incidental in the sense defined above. For the PQ solution, the theoretical problems have been extensively discussed, and we will review some of the issues. The primary focus of this paper will be the challenges to obtaining a plausible implementation of the NB solution. In both PQ and NB, the inadequacies of current proposals concern the structure of the microscopic, ultraviolet theory and particularly the complexity and plausibility of the structures necessary for an effective solution.

1. The principal difficulty with the axion mechanism is that the PQ symmetry needs to be of very high quality. If this symmetry is an accident, it must be a remarkably good one. If the symmetry and its breaking are described by a conventional effective field theory, the required quality can be achieved with a $Z_{N}$ symmetry, but requires $N \geq 11$ or so. This is hardly a compelling explanation for the smallness of an inconsequential parameter of the Standard Model. ${ }^{3}$ In string theory, the situation for light axions appears better, but a solution in this framework requires assumptions about the stabilization of moduli which, while perhaps imaginable, at least at present are impossible to verify. In the string framework, one must also hypothesize an unconventional cosmology and typically some tuning of initial conditions, unless the axion decay constant is surprisingly small.

\footnotetext{
${ }^{1}$ In the interesting alternative case of spontaneous parity violation, models and their criteria for success were discussed in [11-13]. Another mechanism in the case of spontaneous CP violation, distinct from NB, involves the introduction particular "shaping symmetries" in the underlying flavor structure [15].

${ }^{2}$ Other solutions [16-18] possess close similarities to the solutions with approximate U(1)s $[16,17]$ or NB [18].

${ }^{3}$ In [19], the possibility that $N$ is large in order to account for dark matter was considered. It was shown that dark matter can account for a large value of $N$, but not large enough to solve the strong CP problem.
} 
2. As we will elaborate in this paper, the NB mechanism is generically on even weaker theoretical ground. If the implementation is not massively fine-tuned, it requires strong dynamics or supersymmetry (though not necessarily at scales of order a few $\mathrm{TeV})$. Strong dynamics are insufficient to protect small $\bar{\theta}$ in the simplest models, and supersymmetric models require gauge mediation $\left(m_{3 / 2} \ll\right.$ splittings in supermultiplets). In addition, new discrete or gauge symmetries and strong coincidences of scales are necessary, as well as a number of degrees of freedom beyond those required by supersymmetry.

Instead of such speculative exercises, one can hope for an experimental resolution. The discovery of an axion would, needless to say, answer the question. However, a large part of the axion parameter space is currently inaccessible. For the NB solution, there is no similar "smoking gun." While we will argue that gauge mediation is a requirement, the scale need not be particularly low.

This paper is organized as follows. In section 2, we review the basic structure of the fermionic sector of NB models. In section 3, we discuss non-supersymmetric models. If such models contain fundamental scalars, one would expect the scale of $\mathrm{CP}$ violation to be high in order to limit the fine-tuning. However, constraints imposed by dangerous higherdimension couplings require a low scale of $\mathrm{CP}$ violation, implying enormous fine tuning. Although compositeness can explain the required hierarchy, we argue that the simplest models typically fail to retain the necessary NB structure. Setting the fine-tuning issue aside, we discuss the sorts of symmetries which might ensure vanishing $\bar{\theta}$ at tree level, and discuss the dangerous radiative corrections to $\bar{\theta}$ that can arise at one and two loop order. In section 4 we turn to supersymmetry. In theories for which supersymmetry is broken well below some "fundamental" ultraviolet scale (perhaps the Planck, string, or compactification scale), we can pose more sharply the question of what it means for the bare $\theta$ to vanish. We argue that in practice there is a heavy axion, and thus a sense in which the supersymmetric NB and PQ models can be considered as different limiting cases of axion models. We discuss how the expectation value of this axion might be fixed and constraints on couplings of the axion to possible CP-violating sectors. We also note that very simple landscape considerations suggest that vanishing of the "bare $\theta$ " in such frameworks is extremely rare, and these is no obvious anthropic selection effect one might invoke. Finally, we discuss the spontaneous breaking of $C P$ and SUSY and the radiative corrections to $\bar{\theta}$ in supersymmetric models with gravity and gauge mediation. In gravity mediation, corrections are typically large and spoil the NB solution. In gauge mediation, the corrections can be smaller, but there are upper bounds on the ratio of the susy-breaking scale to the scale of $\mathrm{CP}$ violation. In section 6 we summarize and conclude.

\section{The essence of the Nelson-Barr mechanism}

The main challenge in solving the strong $\mathrm{CP}$ problem with spontaneous $\mathrm{CP}$ violation is to understand why

$$
\operatorname{Arg} \operatorname{det} \mathrm{m}_{\mathrm{q}}<10^{-10}
$$


while there is a large phase in the CKM matrix. Nelson [8] and Barr $[9,10]$ obtained the first simple, phenomenologically viable models which achieve this and elucidated the general properties of renormalizable Lagrangians that can exhibit $\operatorname{Arg} \operatorname{det} \mathrm{m}_{\mathrm{q}}=0$ at tree level.

A model with minimal field and symmetry content was obtained by Bento, Branco, and Parada (BBP) [20], and serves as a useful starting point for understanding the properties of the NB mechanism. The BBP model introduces additional charge $\pm 1 / 3 \mathrm{SU}(2)$ singlet quarks $q, \bar{q}$, as well as a set of complex fields $\eta_{a}$ neutral under the SM (we will comment on real fields later). The down-type quark mass terms in the BBP model are given by

$$
\mathcal{L}=\mu \bar{q} q+a_{a f} \eta_{a} \bar{d}_{\bar{f}} q+y_{f \bar{f}} H Q_{f} \bar{d}_{\bar{f}}+\ldots .
$$

The $\eta_{a}$ are assumed to have vevs with relative phases, breaking $\mathrm{CP}^{4}$

At tree level, the Lagrangian in (2.2) automatically gives Arg det $\mathrm{m}_{\mathrm{q}}=0$ for the quark masses. However, it is not the most general renormalizable Lagrangian allowed by the symmetries of the SM. Couplings of the form $\eta_{a} q \bar{q}$ and $H Q \bar{q}$ must be forbidden. Similarly, we might like $\mu$ to be the expectation value of a CP-conserving field, which constrains its interactions with the $\eta_{a}$. Discrete symmetries can provide the necessary structure, and we return to this issue in the next section.

The CKM phase in the SM is generated by integrating out the heavy flavor from (2.2). Defining the $4 \times 4$ quark mass matrix as:

$$
\mathcal{M}=\left(\begin{array}{cc}
\mu & B \\
0 & m_{d}
\end{array}\right) ; m_{d} \equiv y v ; B_{f}=a_{a f} \eta_{a},
$$

we need to diagonalize the matrix

$$
\mathcal{M} \mathcal{M}^{\dagger}=\left(\begin{array}{cc}
\mu^{2}+B B^{\dagger} & B m_{d}^{T} \\
m_{d} B^{\dagger} & m_{d} m_{d}^{T}
\end{array}\right) .
$$

If the left hand corner of this matrix is larger than the other entries, we can integrate out the heavy state, leaving the $3 \times 3$ SM mass matrix:

$$
\left(\left(m_{d} m_{d}^{T}\right)_{i j}-\frac{\left(m_{d}\right)_{i k} B_{k}^{\dagger} B_{\ell}\left(m_{d}^{T}\right)_{\ell j}}{\mu^{2}+B_{f} B_{f}^{\dagger}}\right) .
$$

The diagonalizing matrix is the CKM matrix. Note that this procedure is correct only in the limit $\mu^{2}+\left|B_{f}\right|^{2} \gg m_{d}^{2}$; otherwise, the CKM matrix is not unitary.

Obtaining a large CKM phase strongly constrains the parameters. If there is only one non-vanishing $B_{f}$, or if each $B_{f}$ has the same phase, or if $\mu \gg\left|B_{f}\right|$, then the CKM matrix is real. However, if there are two distinct, non-vanishing $B_{f}$ of comparable magnitude and with a large relative phase, and $\mu \lesssim\left|B_{f}\right|$, there is a non-trivial phase. For example, if

\footnotetext{
${ }^{4}$ In fact, in the original BBP model [20], only a single complex field is introduced with Yukawa couplings $\left(a_{f} \eta+a_{f}^{\prime} \eta^{*}\right) \bar{d}_{\bar{f}} q$. This structure is sufficient as long as $a_{f}$ and $a_{f}^{\prime}$ are nonzero, $a_{f} \neq a_{f}^{\prime}$, and a required discrete symmetry under which $\eta, q$, and $\bar{q}$ transform is a $Z_{2}$ instead of a more general $Z_{N}$. We consider the form of eq. (2.2), with multiple $\eta_{a}$ and vanishing $a_{f a}^{\prime}$, anticipating possible $Z_{N}$ symmetries as well as the extension of the BBP model to supersymmetry.
} 
$B=(0, b, c)$, a phase of order $\operatorname{Im}(\mathrm{b} / \mathrm{c})$ enters the CKM matrix. We see that a rather close coincidence of scales is required between the real and imaginary parts of different fields. The severe challenges for non-susy NB theories will be discussed in the next section.

\section{Nonsupersymmetric Nelson-Barr models}

In this section we consider nonsupersymmetric Nelson-Barr models. We begin with a survey of the basic issues and challenges confronting such models already at tree level, and then elaborate on two of the issues that arise when radiative corrections are included.

\subsection{Basic challenges}

Without supersymmetry, it is a simple matter to construct models of spontaneous CP violation. We can, for example, introduce two real fields, $\sigma$ and $\pi$, the first CP-even and the second CP-odd, with appropriate NB-type couplings to fermions and a potential that leads to a vev for each. Likewise with complex fields it is not difficult to spontaneously break $\mathrm{CP}$, if there is sufficient freedom in the specification of the scalar potential (for a principled discussion of necessary and sufficient conditions, see [21].

However, NB models, to be viable, must confront several theoretical challenges:

1. Further symmetries are necessary to enforce the necessary structure of the mass matrix, even at the renormalizable level. In the BBP model discussed in the previous section, since $\mu \lesssim\left|\left\langle\eta_{a}\right\rangle\right|$, it is necessary suppress or forbid dimension- 4 couplings of the form $\eta_{a} q \bar{q}$. Likewise we must suppress $H Q \bar{q}$. One possibility is to allow the new scalars and fermions to transform under a $Z_{N}$ symmetry (if $N>2$, then the scalars must be complex, as in the model discussed above):

$$
\eta_{a} \rightarrow e^{\frac{2 \pi i k}{N}} \eta_{a}, \quad q_{f} \rightarrow e^{-\frac{2 \pi i k}{N}} q_{f}, \quad \bar{q}_{f} \rightarrow e^{\frac{2 \pi i k}{N}} \bar{q}_{f} .
$$

With other fields neutral, we obtain a Lagrangian of the desired form. It is not difficult to write down models which spontaneously break both $\mathrm{CP}$ and the $Z_{N}$. We will discuss possible gauge symmetries when we consider supersymmetry in the next section.

2. The scale of spontaneous CP breaking $m_{C P}$ should be low compared to the cutoff $\Lambda$. Dimension-5 operators such as

$$
\eta_{a}^{*} \eta_{b} \bar{q} q, \quad \eta_{a} H Q \bar{q}
$$

for example, can induce $\bar{\theta}$ of order $\left(m_{C P} / \Lambda\right)$. Note that the $Z_{N}$ symmetry defined in eq. (3.1) (or possible $\mathrm{U}(1)$ symmetries) does not help to suppress higher-dimension operators like (3.2). Without further symmetries or fine-tuning, even if the cutoff is $\Lambda=M_{p}$, suppression of such operators requires

$$
m_{C P} \lesssim 10^{8} \mathrm{GeV}
$$


3. As in any non-supersymmetric or non-composite model, light scalars are fine-tuned. Here we require at least two such scalars at a scale $m_{C P} \ll M_{p}$, and the fine-tuning of each of these masses is much worse than just fine-tuning $\bar{\theta}$ by itself. It is difficult to make sense of $N B$ models outside of a broader framework in which $m_{C P} / M_{p}$ is naturally small.

4. As we have seen in the previous section, to obtain a substantial CKM angle, it is critical that the expectation values of different $\mathrm{CP}$-odd and $\mathrm{CP}$-even fields (times suitable couplings) coincide to better than an order of magnitude.

5. We might want to account for $\mu$ dynamically, i.e. through the expectation value of a fundamental or composite field $S$. Additional symmetries need to be introduced to avoid inducing phases in $S$ from couplings of $S$ to the $\eta_{a}$.

6. Even when it vanishes at tree-level, $\bar{\theta}$ is often generated radiatively at the scale $m_{C P}$.

Loop effects are particularly problematic. They cannot be suppressed simply by additional (bosonic) symmetries or by lowering the scale of CP violation. These corrections will be the subject of the next section.

\subsection{Radiative corrections to $\theta$ in non-supersymmetric theories}

Even if one closes one's eyes to fine tunings, and one is willing to accept a low scale for $\mathrm{CP}$ violation, loop corrections are quite problematic in NB models. Threshold corrections to $\bar{\theta}$ have to be considered on a model-by-model basis, but certain operators are typically problematic. BBP studied $\bar{\theta}$ at one loop in [20]. Below, we review and reinterpret their result, and observe further problematic contributions at two loop order. We will see that the one loop sensitivity of $m_{C P}$ to the UV cutoff requires us to add structure, such as supersymmetry or a dynamical origin for the scalars, and then to consider all of the other issues in that larger framework. In the subsequent section we discuss composite models and see that while the fine-tuning of $m_{C P}$ can be resolved, simple cases will either have difficulty maintaining $\bar{\theta}=0$ at tree level, or will have one loop corrections to $\bar{\theta}$ similar to non-composite models. This will lead us to consider NB in the supersymmetric context.

In the BBP model, dangerous contributions to $\bar{\theta}$ arise at one loop from the Higgs portal operators

$$
\left(\gamma_{i j} \eta_{i}^{\dagger} \eta_{j}+\lambda_{i j} \eta_{i} \eta_{j}+c c\right) H^{\dagger} H
$$

$\lambda_{i j}$ can be forbidden by a $Z_{N}$ symmetry with $N>2$, so we consider the effects of $\gamma_{i j}$. Unless the $\gamma_{\mathrm{s}}$ are very small, these couplings make a large contribution to the Higgs mass. In the context of a solution to the $m_{C P}$ hierarchy problem, there might or might not be a principled reason why the couplings are small, but a priori they indicate only another contribution of many to the tuning of $m_{H}^{2}$. At one loop, the diagram of figure 1 gives a complex correction to the SM down-type Yukawa coupling, contributing to a shift in $\bar{\theta}$ of order

$$
\Delta \bar{\theta} \simeq \operatorname{Im} \operatorname{Tr} y^{-1} \Delta y \simeq \frac{\eta_{a} a_{a f} a_{b f} \gamma_{b c} \eta_{c}^{*}}{16 \pi^{2} m_{C P}^{2}} .
$$

Adequately suppressing $\bar{\theta}$ requires the $a$ and/or $\gamma$ couplings to be small. 


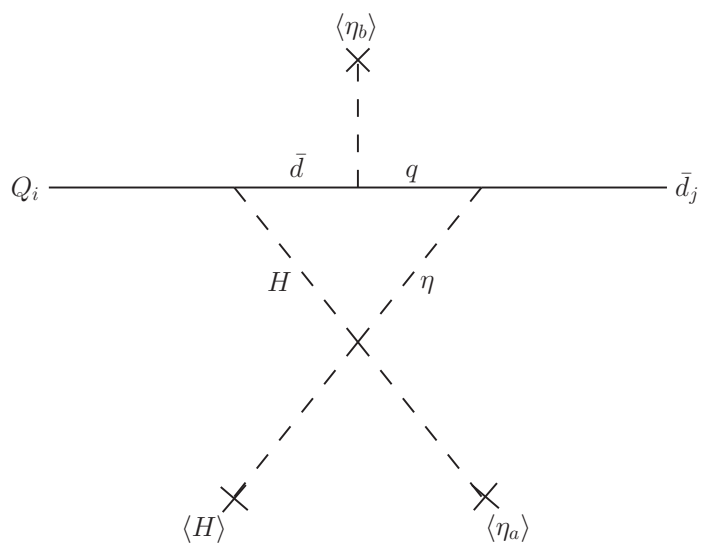

Figure 1. Example threshold correction to Arg det $m_{d}$.

The authors of [20] took the viewpoint that whatever solves the SM hierarchy problem might suppress the portal couplings. Such suppressions can occur in supersymmetric or composite theories (both of which solve the $m_{C P}^{2}$ hierarchy problem, but not necessarily the full $m_{H}^{2}$ one). These theories involve significant extra structure beyond the minimal BBP model, and the radiative corrections to $\bar{\theta}$ must be considered in the full theories. Without supersymmetry or extra dynamics, the Higgs mass is simply tuned, and small $\theta$ is problematic.

At two loop order, there are additional contributions which must be suppressed. In particular, insertions of the operator

$$
\mathcal{L}_{\eta^{4}}=\gamma_{i j k l} \eta_{i} \eta_{j} \eta_{k}^{*} \eta_{\ell}^{*}
$$

can contribute phases to the operators $\mu \bar{q} q$ and $Q H \bar{d}$. The relevant Feynman diagrams contain a loop of gauge bosons and an $\eta$ loop, with insertions of $\mathcal{L}_{\eta^{4}}$; an example is given in figure 2 (this contribution is similar to the "dead duck" graph noted in [8]). The contribution to $\bar{\theta}$ is of order

$$
\Delta \bar{\theta} \simeq \frac{g^{2} a_{a f} a_{c f} \eta_{b}^{*} \eta_{d} \gamma_{a b c d}}{\left(16 \pi^{2}\right)^{2} m_{C P}^{2}}
$$

Again, unless the couplings are surprisingly small, the correction is several orders of magnitude to large. In the supersymmetric case, we will see that these contributions can be suppressed, but new issues will arise.

\subsection{Models with strong dynamics}

The low scale of $\mathrm{CP}$ violation may be protected by strong dynamics. For example, the CP-odd scalars could be pseudogoldstone mesons $\Pi$ of an $\mathrm{SU}(\mathrm{N})$ gauge theory in which condensates spontaneously break approximate chiral flavor symmetries,

$$
\left\langle\bar{\psi}_{i} \psi_{j}\right\rangle=B f_{\Pi}^{2} \exp \left(i \Pi^{a} t^{a} / f_{\Pi}\right)
$$




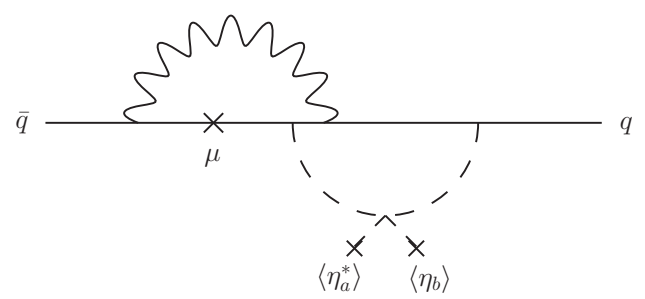

Figure 2. Example two-loop contribution to the phase of $\mu$.

in analogy with the pions of $\mathrm{QCD}$. The $\Pi$ fields can obtain nonzero vevs naturally from a particular pattern of chiral symmetry breaking (as in, e.g., Dashen's model [22]). In this case, BBP-type couplings to the Standard Model and the $q, \bar{q}$ messengers (assumed for now to be fundamental fermions) might arise from higher-dimensional operators of the form

$$
\frac{1}{\Lambda^{2}} \kappa_{i j}^{f} \bar{\psi}_{i} \psi_{j} \bar{d}_{f} q / \Lambda^{2} \rightarrow B \frac{f_{\Pi}^{2}}{\Lambda^{2}} \operatorname{Tr}\left[\kappa^{f} e^{i \Pi^{a} t^{a} / f_{\Pi}}\right] \bar{d}_{f} q+\ldots
$$

If the hierarchy between the scale of the gauge theory $\sim f_{\Pi}$ and the UV cutoff $\Lambda$ is large, the effective couplings $a_{a f}$ in eq. (2.2) may be very small, and the effective scale of CP violation much smaller than $f_{\Pi}$. We can see from the form of eq. (2.5) that the CKM phase can still be large if $\mu$ is sufficiently small. Furthermore, the one loop BBP radiative correction - generated here by couplings of the form $H^{\dagger} H \bar{\psi} \psi / \Lambda$ - is suppressed when the effective $a_{a f}$ couplings are small.

Unlike in the fundamental scalar case, however, it is difficult to implement discrete symmetries needed to keep $\mu$ real. Permitting (3.9) while forbidding the similar 4 -fermi operator $\bar{\psi} \psi \bar{q} q$ requires the discrete symmetry to act chirally on $\psi, \bar{\psi}$ (and, for example, on $q, \bar{q}$ ), but explicit chiral symmetry breaking is necessary to generate the spontaneous CPV potential when the CP-odd scalars are pseudogoldstones. This breaking might be soft, as in a set of masses $m$ for the $\psi, \bar{\psi}$, and thus the coefficient of $\bar{\psi} \psi \bar{q} q / \Lambda^{2}$ might be suppressed by $m / \Lambda$. But if $m$ is not too different from $f_{\Pi}$, then $f_{\Pi} / \Lambda$ must be less than $10^{-10}$, resulting in an unacceptably low value for $m_{C P}$.

It is even more difficult to understand the NB structure and the reality of the effective $\mu$ if the messenger fields $q, \bar{q}$ are baryons of the gauge theory. In this case the baryon mass is expected to arise principally from spontaneous chiral symmetry breaking, which by construction breaks CP.

We stress that it is not impossible to build NB-type models with strong dynamics, but it requires more complicated structures. A minimal example was constructed in ref. [23], consisting of a BBP-type model in which the $\bar{\psi} \psi \bar{q} q$ operator is forbidden by a gauged subgroup of the chiral flavor symmetry. This symmetry might also be discrete. The Dashen mass terms are forbidden by the symmetry, but the potential can still break CP with suitable dimension-6 operators $(\bar{\psi} \psi)^{2}$. Ref. [23] also showed that models with acceptably small radiative corrections to $\bar{\theta}$ could be distinguished by the flavor transformation properties of the CPV spurions present in the low-energy theory. BBP-type models with generic couplings possess CPV spurions in the infrared in both the fundamental and anti-fundamental 
representations of $\mathrm{SU}(3)_{d}$, and as such they fail the criteria of [23]. This is reflected in the large one loop correction to $\bar{\theta}$. However, when the couplings $a_{a f}$ are small, as can arise in strongly-coupled models as discussed above, the low-energy theory contains only an $\mathrm{SU}(3)_{d}$-fundamental spurion and the criteria for small corrections to $\bar{\theta}$ are met.

\section{$4 \mathrm{CP}$ in supersymmetric theories: axions, moduli, and $\theta$ at tree level}

Supersymmetry, with SUSY breaking at scales well below the scale of CP violation, can significantly ameliorate the Nelson-Barr fine-tuning problem. In addition, SUSY can forbid some of the problematic higher-dimension operators and quantum corrections to $\bar{\theta}$ encountered in the non-SUSY case. In this section, we consider supersymmetric NelsonBarr models and their symmetries. We first review some of the problematic aspects of the Peccei-Quinn solution of the strong CP problem and their possible resolution. Then we consider more carefully the underlying premise that $\mathrm{CP}$ can naturally be a good symmetry, and as a result that the bare $\bar{\theta}$ vanishes. In both cases the questions are ultraviolet-sensitive and the resolutions depend on the structure of the microscopic theory. In particular, if there is an underlying landscape, small bare $\bar{\theta}$ is implausible.

We first review some aspects of the axion solution, with and without supersymmetry. The most challenging aspect of the Peccei-Quinn solution of the strong CP problem is understanding why the global symmetry is so good. Global symmetries should arise only as accidents of gauge symmetry and the structure of low dimension terms in an effective action. It was quickly recognized that this is a challenge for the PQ mechanism [24]. From a PQ-violating potential $V_{p q v}$, we can define an axion quality factor,

$$
Q_{a} \equiv \frac{f_{a} \frac{\partial V_{p q v}(a)}{\partial a}}{m_{\pi}^{2} f_{\pi}^{2}} .
$$

Solving the strong $\mathrm{CP}$ problem requires

$$
Q_{a}<10^{-10}
$$

In a conventional effective field theory analysis (i.e. finite number of degrees of freedom above $f_{a}$ ), small $Q_{a}$ is highly non-generic. If the axion arises as the phase of a field $\Phi$,

$$
\langle\Phi\rangle=f_{a} e^{i a / f_{a}},
$$

symmetry violating operators like

$$
\frac{\Phi^{n+4}}{M_{p}^{n}}
$$

spoil the PQ mechanism even for $f_{a}=10^{11} \mathrm{GeV}$ unless $n>7$. Such suppression can be obtained with a discrete $Z_{N}$ symmetry, with $N \geq 11$, but such a model appears contrived.

Witten pointed out early on that string theory provides a possible resolution to the problem of the quality of the PQ symmetry [25]. This is most easily understood in the 
framework of supersymmetry. Typically string models possess moduli, $\Phi$, whose imaginary component obeys a discrete shift symmetry:

$$
\Phi=x+i a ; \quad a \rightarrow a+2 \pi .
$$

This symmetry guarantees that any superpotential is a function of $e^{-\Phi}$ at large $x$. Here $x$ might be $\frac{8 \pi^{2}}{g^{2}}$, for some gauge coupling $g$.

In this setting, the primary question is why the theory sits in an asymptotic region of the moduli space where $e^{-x}$ is very small. It is consistent at least with the fact that the observed gauge couplings are small, but a detailed connection is not possible at present, much less reliable computations [26].

We turn now to theories where CP is a symmetry of the microscopic dynamics. Here we can make a connection with string axions discussed above. In known string theories, CP is a good symmetry [27-29]. For typical string compactifications, this statement means that there is a subspace of the moduli space on which CP is conserved, and CP is spontaneously broken on the rest. In supersymmetric theories, the moduli fields include both a CP-even and a CP-odd scalar, as in eq. (4.5), and we will refer to them as saxions $x_{i}$ and axions $a_{i}$, respectively. We can define $a_{i}=0$ as the $\mathrm{CP}$ conserving point. $\mathrm{CP}$ is spontaneously broken if some of these axions are stabilized at $a_{i} \neq 0$. Generally one or moduli couple to each of the gauge groups in the classical theory, providing candidate axions. The question of whether there is a non-zero $\theta$ is then a question of whether the relevant axions are heavy and fixed at $\mathrm{CP}$ conserving points.

If the moduli are stabilized supersymmetrically, the CP-even and CP-odd states are fixed together. Suppose that we have a single modulus, with

$$
W=-\alpha e^{-\Phi / b}+W_{0} ; \quad K=-\log \left(\Phi+\Phi^{\dagger}\right),
$$

with $W_{0}$ small, as in the KKLT scenario [30]. Then

$$
\Phi \approx b \log \left(W_{0} / \alpha\right) .
$$

Provided $W_{0}$ and $\alpha$ are real, $\Phi$ is real. If $\Phi$ couples to the QCD gauge fields as $\Phi W_{\alpha}^{2}$, it generates no tree-level contribution to $\theta$. Plausibly, if $W_{0}$ is large, CP remains unbroken, and $\Phi$ is very heavy.

Should $W_{0}$ be real? If we assume $W_{0}$ results from CP-conserving dynamics, it is automatically real. On the other hand, flux landscapes provide a model where complex $W_{0}$ appears more likely. In such cases $W_{0}$ is the sum of many contributions associated with many different fluxes, of which we expect about half to be CP-even and half to be CP-odd. $\mathrm{CP}$ preservation amounts to requiring half of the of the fluxes to vanish. In other words, given $10^{500}$ states, only $10^{250}$ conserve $\mathrm{CP}$ and have vanishing $W_{0}$, and correspondingly $\mathrm{CP}$-conservation appears very non-generic. Moreover, as noted earlier, it is hard to see what might select for small $\theta$. However, absent a sharp UV prediction for $W_{0}$, we can simply take its reality as a requirement of the NB setup.

We can ask what may happen when we introduce a sector in which CP is spontaneously broken with characteristic scale $\mu$. If this sector does not break supersymmetry, we might 
expect additional, CP-violating terms in the superpotential of order $\mu^{3} e^{-S}$. These terms will shift the minimum of the axion field, but their contribution is suppressed if $b$ is large. If, for example, $e^{-S}<10^{-15}$ and $b=5$, then $\theta<10^{-12}$. Alternatively, if $b=1$, the contribution to $\theta$ is suppressed by at least ten order orders of magnitude provided the scale $\mu$ is at least three orders of magnitude below $M_{p}$. In non-supersymmetric models (e.g. cases where the scale of SUSY-breaking is $\gg \mu$ ) with axions, one would expect the difficulties to be at least as severe; it is not clear in such contexts that terms violating the Peccei-Quinn symmetry must be exponentially small.

The assumption that $W_{0}$ is real constrains a combination of the supersymmetry breaking and $\mathrm{CP}$ violating scales. In particular, we might expect $\mathrm{CP}$ violation to generation a complex term in the superpotential, $W_{0} \sim \mu_{C P}^{3}$. If there is no suppression of the phase, the requirement of cancellation of the cosmological constant yields the constraint:

$$
\mu_{C P}^{3}<M_{3 / 2} M_{p}^{2}
$$

\section{SUSY Nelson-Barr models}

In this section, we assume that any would-be axions are massive and fixed in a CP conserving manner. We then ask what are the requirements on SUSY NB models required to account for a very small $\bar{\theta}$. The Lagrangian of (2.2) naturally extends to a superpotential:

$$
W=\mu \bar{q} q+\lambda_{a f} \eta_{a} q \bar{d}_{f}+y_{f \bar{f}} H_{d} Q_{f} \bar{d}_{f}+\ldots .
$$

For the moment we continue to treat $\mu$ as a dimensionful constant. While the absence of undesirable renormalizable interactions like $\eta q \bar{q}$ and $H_{d} Q \bar{q}$ can be technically natural due to nonrenormalization theorems, they can be forbidden in a more principled way with, for example, discrete symmetries like (3.1). Again a coincidence in scales among the $\eta_{a}$ vevs is required, as well as $\mu \lesssim\left|\lambda_{a f} \eta_{a}\right|$.

As emphasized above, putting NB into a larger and more natural framework incurs new challenges. The prime example in SUSY models is that the $\eta_{a}$ must be sequestered from the supersymmetry breaking sector to avoid, e.g., giving phases to the gluino mass, among other problems [31]. We might expect the SUSY breaking theory to exhibit either an exact (discrete) $R$ symmetry, or at least approximate accidental one. If there is an identifiable Goldstino field, $Z$ (assumed chiral), then couplings of the $\eta_{a}$ to $Z$ must be suppressed.

Replacing $\mu$ by a dynamical field $S$ may be desirable and requires further symmetries. For example, it is critical to forbid renormalizable couplings between $S$ and the $\eta_{a}$.

\subsection{Breaking of CP and $Z_{N}$ in SUSY}

If $\mathrm{CP}$ is violated at or below the scale of supersymmetry breaking, the low-energy theory can be studied in the non-supersymmetric framework of the previous section. Therefore, we focus on $\mathrm{CP}$ violation at scales much higher than those of supersymmetry breaking. We will not attempt to be exhaustive, but we consider models that illustrate some of the challenges. We consider two classes of models:

1. Models in which the $\mathrm{CP}$ violating fields are fixed supersymmetrically. Here there is a discrete set of vacua and all fields have mass of order the scale of $\mathrm{CP}$ violation. 
2. Models in which the CP violating fields are fixed by SUSY breaking dynamics. We take the scale of CP violation to be much larger than the scale of SUSY breaking; in this situation, $\mathrm{CP}$ is broken by fields in approximate flat directions.

\subsubsection{CP broken by supersymmetry-conserving dynamics}

To write a simple model that breaks $C P$ in isolated vacua, we introduce two fields $\eta_{1}$ and $\eta_{2}$, odd under a $Z_{2}$ symmetry, and fields $X$ and $Y$ that are even. We can also suppose an $R$ symmetry (for simplicity we will take it to be continuous, but it can also be a discrete subgroup) under which $X$ and $Y$ have $R$ charge 2 and the $\eta_{i}$ are neutral. Then we can take the superpotential to have the form, without loss of generality:

$$
W=X \mu^{2}+X\left(a \eta_{1}^{2}+b \eta_{1} \eta_{2}+c \eta_{2}^{2}\right)+Y\left(a^{\prime} \eta_{1}^{2}+b^{\prime} \eta_{1} \eta_{2}+c^{\prime} \eta_{2}^{2}\right)
$$

This superpotential typically has minima in which $\eta_{1}$ and $\eta_{2}$ have phases, breaking CP. If $q, \bar{q}$ are both odd under the $Z_{2}$, with $R$ charge 1 , and $\bar{d}_{f}$ is even, with $R$ charge 1 , then we obtain the NB superpotential at the renormalizable level.

There are a number of issues with models of this type. In particular, if supersymmetry breaking is associated with a Goldstino superfield in a hidden sector, $Z$, these symmetries will not forbid $Z \eta_{1} \eta_{2}$ couplings, leading to $\mathrm{CP}$ violating phases in ordinary soft breaking terms. $Z_{N}$ symmetries with larger $N$, while forbidding these couplings, require more structure in order to obtain a superpotential that is both $Z_{N}$ invariant and spontaneously breaks $\mathrm{CP}$ (and $Z_{N}$ ).

Another model for spontaneous CP violation has been presented in [32]. In addition to a discrete symmetry, the model relies on a continuous global symmetry to suppress couplings which would induce $\theta$ at tree level. If the $\mathrm{U}(1)$ is replaced by a discrete subgroup, at least a $Z_{3} \times Z_{5}$ symmetry is needed to suppress dangerous renormalizable operators.

\subsubsection{Theories with flat directions}

String theory constructions suggest another possibility which can lead rather naturally to the NB structure. There are two elements. First, string models often possess U(1) symmetries beyond those of the Standard Model, as well as additional fields, which can yield the required superpotential for the NB models. Second, there are often approximate flat directions in which CP-odd fields can obtain large expectation values. Under suitable conditions, these vevs may spontaneously break $\mathrm{CP}$.

In particular, the gauge group $E_{6}$, familiar in Calabi-Yau compactifications of the heterotic string, suggests the possibility of two additional U(1)s at some energy scale as well as several additional fields. In terms of $O(10) \times \mathrm{U}(1) \subset E_{6}$, the 27 of $E_{6}$ decomposes as

$$
27=16_{-1 / 2}+10_{1}+1_{-2} .
$$

We will treat the theory as if this symmetry is broken to the Standard Model $\times \mathrm{U}(1) \times \mathrm{U}(1)$. Then we can list the fields and their charges under the two U(1)s:

$$
\begin{aligned}
& Q, \bar{e}, \bar{u}=(-1 / 2,1) ; \quad L, \bar{d}=(-1 / 2,-3) ; \quad \bar{q}=(1,2) ; \quad q=(1,-2) ; \quad \eta=(-1 / 2,5) ; \\
& H=(1,2) \quad \bar{H}=(1,-2) \quad S=(-2,0) .
\end{aligned}
$$


Note that the $\eta$ is essentially the right-handed neutrino of $O(10)$, while the $S$ is the field in $E_{6}$ outside of the 16 or $10 . q, \bar{q}$, and $\ell, \bar{\ell}$ arise from the 10 of $O(10)$. Anomaly cancellation is readily satisfied by including an additional $q, \bar{q}, \ell, \bar{\ell}, \eta, S$ for each generation. In addition, we assume that there is one additional $S, \bar{S}$ pair and one additional $\eta, \bar{\eta}$ pair (and allow the possibility of other incomplete multiplets, particularly for the Higgs field).

With these charge assignments, the most general cubic superpotential involving $S, \eta, q$, $\bar{q}$ and the ordinary matter fields is precisely that of eq. (5.1). Moreover, at the renormalizable level, the classical theory possesses flat directions with non-zero $\eta_{i}, \bar{\eta}, S_{i}, \bar{S}$.

The flat directions may be lifted by supersymmetry-breaking effects and dimension- 5 operators. If some of the soft masses in the flat directions are negative, some of the fields will receive large expectation values. If there are quartic superpotential couplings, e.g. $\frac{1}{M_{p}} \eta_{i} \eta_{j} \bar{\eta}^{2}$ and $\frac{1}{M_{p}} S_{i} S_{j} \bar{S}^{2}$, then these expectation values are of the order

$$
S^{2}, \eta^{2} \sim m_{\text {susy }} M_{p}
$$

With several fields, there will typically be $\mathrm{CP}$ violating minima of the potential.

Many problematic higher-dimension operators are forbidden by holomorphy and the $\mathrm{U}(1)$ s. However, a surviving class of dimension-5 operators, $S_{i} \bar{S} \eta_{j} \bar{\eta}$, must be forbidden to avoid large phases in $S$. These couplings can be forbidden by discrete symmetries. One virtue of this type of model is that it is compatible with the existence of a (discrete) $R$ symmetry, which can suppress couplings of the $\eta$ fields to any would-be supersymmetrybreaking sector and possible messengers.

Another potential difficulty is the large size of the $\eta_{i}$ expectation values. These are sufficiently large that, depending on the scale of supersymmetry breaking and the suppression scale, they have the potential to induce $\bar{\theta}$ through dimension- 6 operators.

\subsection{Breaking of supersymmetry}

We have already noted that supersymmetry breaking introduces new potential contributions to $\bar{\theta}$. Many of these contributions do not decouple, even as the supersymmetry breaking scale is taken arbitarily large. As a result, a successful supersymmetric solution to strong CP requires suppression of phases in the gluino mass, as well as a high degree of degeneracy, proportionality, and suppression of phases in squark masses and $A$-terms [31], regardless of the scale of supersymmetry breaking.

We distinguish two classes of models: those, like gravity-mediated models, where the soft breaking terms of the SM fields are of order $m_{3 / 2}$, and those, like gauge mediated models, where $m_{3 / 2}$ is parametrically smaller.

Consider first gravity-mediated models. In these models, one general issue is $\langle W\rangle \sim$ $m_{3 / 2} M_{p}^{2}$. If $\langle W\rangle$ is complex, this feeds into $\theta$ through phases, for example, at one loop in the gaugino mass (this is the familiar anomaly-mediated contribution). In section 4, we raised general questions about the reality of $\langle W\rangle$, and argued that in flux landscapes, at least, real $\langle W\rangle$ is unlikely. More generally, apart from some sort of anthropic selection, no convincing mechanism has been put forward to account for the value of the cosmological constant. So the failure of landscape models to account for small phases is troubling. 
In gauge-mediated models, the situation can be significantly better. Comparing the anomaly-mediated to the gauge-mediated gluino mass, we require

$$
\frac{\alpha_{s}}{4 \pi} \frac{m_{3 / 2}}{m_{\text {susy }}}<10^{-10} .
$$

This constraint places a loose upper bound on the underlying scale of supersymmetry breaking if $W$ possesses an order one phase.

In both gravity and gauge mediation, there may be other strong constraints, depending on the nature of supersymmetry breaking. If supersymmetry is broken in a hidden sector through a gauge-singlet chiral field, $Z$, with $F_{Z}=f$, then any phase in $f$ can feed into soft breaking terms, yielding phases for the gluino, for example, as well as squark mass matrices. These, in turn, contribute to $\theta$. In the models we have studied, these might arise from couplings such as

$$
W_{\eta-Z}=\lambda \eta_{i} \eta_{j} Z
$$

at dimension three in $W$, or even through terms of dimension 2. Such undesirable terms can be forbidden if $Z$ is charged under some symmetry (as in some models of dynamical supersymmetry breaking), or by combinations of continuous and discrete symmetries in the models of CP breaking by pseudomoduli of the sort discussed in the previous section. For example, couplings of combinations like $\eta_{i} \bar{\eta}$ to $Z$ can be forbidden by $R$ symmetries. In the models with discrete vacua, this problem is more challenging. In gauge-mediated models, it is also necessary to forbid couplings of the $\eta$ fields to messengers. This can again arise from the $R$ symmetries consistent with the flat direction models.

If non-renormalizable terms coupling CP-breaking fields to $Z$ are permitted by symmetries, these will constrain the scale of $\mathrm{CP}$ violation. Certain Kahler potential terms are difficult to suppress by symmetries. However, one can contemplate higher scales of CP violation than in the non-supersymmetric case.

Overall, then, both in gravity and gauge mediation, it appears possible to avoid dangerous new sources of phases at tree level, without large arrays of new fields or excessively complicated new symmetry structures. Gravity mediation requires stronger constraints on the reality of $W$.

\subsection{Loop corrections in supersymmetric theories}

Supersymmetric theories are immunized against many of the types of corrections found in non-supersymmetric theories as a consequence of holomorphy and non-renormalizations. In particular, large terms of the form $H^{*} H \eta_{i}^{*} \eta_{j}$ and $\eta_{i} \eta_{j} \eta_{k}^{*} \eta_{l}^{*}$ need not arise (the corresponding superpotential terms can be suppressed by symmetries and the smallness of the $\mu$ term). There are, however, new possible sources of corrections to $\theta$. We divide our discussion between gravity mediated and gauge mediated models. Loop corrections in gravity mediated models, as discussed in [31], are quite problematic. Gauge mediated models are better controlled [18].

We assume that tree level contributions to phases of gaugino masses are highly suppressed. Beyond this, we require, as discussed above, suppression of phases in the underlying supersymmetry breaking $f$ term and the superpotential. But there are still potential 
difficulties. As discussed in [31], already at one loop, there are contributions to gaugino masses arising from loops involving heavy fields in the $\mathrm{CP}$ violating sector. In the simplest model, the heavy field is a Dirac particle, of mass $m_{D}$, consisting of a charge $1 / 3$ field,

$$
\bar{D}=\sum B_{f} \bar{d}_{f}+\mu \bar{q}
$$

and a field of charge $-1 / 3, D=q$. There is a soft breaking term,

$$
\mathcal{L}_{q \bar{D}}=A_{D} m_{D} \bar{D} D
$$

The gluino mass receives contributions proportional to $A_{D}^{*}$. In general, there is no reason for the phase of $A$ to vanish; this requires a very specific alignment of expectation values and couplings. It could arise in the presence of an SU(4) symmetry acting on $\bar{d}$ and $\bar{q}-$ something clearly not present in this structure. The phase must be smaller than $10^{-8}$ or so. Similarly, there are potential contributions proportional to $F_{\eta_{a}}$. In supergravity models, these may naturally be suppressed by $\left(m_{3 / 2} / M_{p}\right)^{1 / 2}$, so they become problematic if the scale of supersymmetry breaking is greater than $10^{4} \mathrm{GeV}$ or so.

As discussed in [31], there are additional contributions arising from phases in soft scalar mass terms. Suppressing these requires a remarkably high degree of degeneracy and proportionality. Overall, then, there is a set of issues similar to, but more severe than, the usual flavor problems of supergravity theories.

Gauge mediated models are characterized by features which ameliorate the problems noted above. ${ }^{5}$ First and foremost, new sources of flavor violation are absent, and $A$ terms are highly suppressed.

In addition, insertions of $F_{\eta_{a}}$, which also enter in loop corrections to gaugino masses, are small if SUSY breaking does not couple to the $\eta_{a}$ at tree level. SUSY-breaking $F$-terms for the $\eta_{a}$ are generated radiatively from Kahler potential operators such as $Z^{\dagger} Z \eta_{a}^{\dagger} \eta_{b} / m_{C P}^{2}$, but in the minimal model they appear only at three loop order. These statements need not hold in theories where messengers mix with other fields so as to gain large $A$ terms, or where there are " $\mu$-terms" for some of the $\eta$ fields.

At higher loop order, complex A-terms and flavor-violating soft masses can be generated in gauge mediation. Such terms can give a weak upper bound on the hierarchy $F_{Z} / m_{C P}^{2}$. For example, in minimal gauge mediation, a Kahler potential operator of the form $Z^{\dagger} Z q \bar{d}_{f} \eta_{a} / m_{C P}^{3}$ is generated at 3-loop order from loops of the $\eta$ fields connected to ordinary gauge mediation loops. This operator provides a phase to the gluino mass in a manner similar to a complex A-term of the form $A_{\gamma} \eta q \bar{d}$ (although the operator involves heavy fields and cannot be written as an A-term at the scale $m_{C P}$ ). Because of the high loop suppression, the bound from $\bar{\theta}$ is weak: $F_{Z} / m_{C P}^{2} \lesssim 10^{-2}$.

Furthermore, all non-minimal flavor violation among the light fields comes from the coupling $a_{a f} \eta_{a} \bar{d}_{f} q$ and the mixing of light right-handed fields with $a_{a f}\left\langle\eta_{a}\right\rangle \bar{d}_{f}$. If $\mu \ll$ $a_{a f}\left\langle\eta_{a}\right\rangle$, the light field is mostly $\bar{q}$, and the mixing is small. Since $\mu \gg a_{a f}\left\langle\eta_{a}\right\rangle$ is in conflict with the large CKM phase, and there is no obvious reason for the scales to be coincident, contributions to $\bar{\theta}$ in gauge-mediated NB models can be even further suppressed by $\mu / m_{C P}$.

\footnotetext{
${ }^{5}$ See also the discussion in [32] for the possibility of suppression through alignment.
} 


\section{Conclusions}

We have argued that solving the strong CP problem is not necessarily an arena for model building cleverness; rather, ideally, the smallness of an inconsequential parameter should emerge as a consequence of features of a theory which explains a range of other phenomena. No currently known model for solving strong CP is completely satisfactory from this point of view.

The shortcomings of the axion solution are well-known. Perhaps the most credible realization is in string theory, where plausible assumptions about moduli fixing may lead to a solution, albeit with a relatively high-scale axion.

In the case of the Nelson-Barr solution, we have argued that non-supersymmetric models are at best very complicated, with intricate symmetries required to suppress higherdimension operators. If these operators are simply suppressed by a low scale of CP violation, models without strong dynamics or supersymmetry require a degree of fine-tuning higher than if $\bar{\theta}$ were simply set to zero by hand. Furthermore, we have argued that dynamical models based on vevs for pseudo-Goldstones are nontrivial to construct. Loop corrections in generic non-SUSY models are even more problematic, making further demands on the theories.

Supersymmetric Nelson-Barr fares somewhat better. Coincidences of scales are still required, but light scalars can be technically natural, and holomorphy greatly restricts the higher-dimension operators that can contribute to $\bar{\theta}$. We described a specific structure in which the NB mechanism is operative and $\mathrm{CP}$ is broken in approximate flat directions by fields carrying new gauge symmetries. Additional discrete symmetries can suppress dangerous couplings of the $\mathrm{CP}$-violating fields to the hidden sector fields and also couplings to messengers. Loop corrections are known to be highly problematic in generic gravitymediated models, but in gauge-mediated models, these effects are under control. So supersymmetric models with additional symmetries and gauge mediation provide a setting in which the Nelson-Barr mechanism is plausible, at least as viewed at relatively low scales.

We have also studied the underlying premise of models that aim to solve the strong CP problem through spontaneous $\mathrm{CP}$ violation: that in such theories, the bare $\theta$ parameter naturally vanishes. We stressed that this is a question of the nature of the ultraviolet theory. In string theory, the value of $\theta$ is generally controlled by the value of an axion field, so the basic assumption is that there are massive axions whose expectation values conserve CP. Perhaps most problematic for the idea of small $\theta$, however, is the possibility of a landscape. We noted that in flux landscapes, in particular, where the heavy axion expectation value is determined by superpotential parameters, these parameters are likely to be complex in an overwhelming majority of states.

So the current status of the strong CP problem can be described by saying we possess three solutions, each with significant flaws. The reader is free to develop his or her own view as to which solution, is any, is most plausible. Unless there are systematic problems with lattice computations which are common to disparate approaches to QCD, the light $u$ quark solution is ruled out. The axion solution requires either very complicated symmetry structures, or some assumptions about moduli stabilization and an unconventional 
cosmological history. The spontaneous $\mathrm{CP}$ solution requires supersymmetry, a variety of additional symmetries, something like gauge mediation, and, perhaps most problematic, an explanation of why moduli are stabilized in a CP-conserving way.

\section{Acknowledgments}

This work was supported by the U.S. Department of Energy grant number DE-FG0204ER41286. We appreciate conversations with Nima Arkani-Hamed, Nathaniel Craig, Ravi Kuchimanci, Anson Hook, Nathan Seiberg, Goran Senjanovic, Scott Thomas, and Luca Vecchi.

Open Access. This article is distributed under the terms of the Creative Commons Attribution License (CC-BY 4.0), which permits any use, distribution and reproduction in any medium, provided the original author(s) and source are credited.

\section{References}

[1] R.D. Peccei and H.R. Quinn, CP Conservation in the Presence of Instantons, Phys. Rev. Lett. 38 (1977) 1440 [INSPIRE].

[2] R.D. Peccei and H.R. Quinn, Constraints Imposed by CP Conservation in the Presence of Instantons, Phys. Rev. D 16 (1977) 1791 [InSPIRE].

[3] M.A.B. Beg and H.S. Tsao, Strong P, T Noninvariances in a Superweak Theory, Phys. Rev. Lett. 41 (1978) 278 [INSPIRE].

[4] H. Georgi, A Model of Soft CP Violation, Hadronic J. 1 (1978) 155.

[5] R.N. Mohapatra and G. Senjanović, Natural Suppression of Strong $p$ and $t$ Noninvariance, Phys. Lett. B 79 (1978) 283 [InSPIRE].

[6] G. Segre and H.A. Weldon, Natural Suppression of Strong $P$ and T Violations and Calculable Mixing Angles in $\mathrm{SU}(2) \times \mathrm{U}(1)$, Phys. Rev. Lett. 42 (1979) 1191 [INSPIRE].

[7] S.M. Barr and P. Langacker, A Superweak Gauge Theory of CP Violation, Phys. Rev. Lett. 42 (1979) 1654 [INSPIRE].

[8] A.E. Nelson, Naturally Weak CP-violation, Phys. Lett. B 136 (1984) 387 [InSPIRE].

[9] S.M. Barr, Solving the Strong CP Problem Without the Peccei-Quinn Symmetry, Phys. Rev. Lett. 53 (1984) 329 [INSPIRE].

[10] S.M. Barr, A Natural Class of Nonpeccei-quinn Models, Phys. Rev. D 30 (1984) 1805 [INSPIRE].

[11] K.S. Babu and R.N. Mohapatra, A Solution to the Strong CP Problem Without an Axion, Phys. Rev. D 41 (1990) 1286 [INSPIRE].

[12] S.M. Barr, D. Chang and G. Senjanović, Strong CP problem and parity, Phys. Rev. Lett. 67 (1991) 2765 [INSPIRE].

[13] R. Kuchimanchi, P/CP Conserving CP/P Violation Solves Strong CP Problem, Phys. Rev. D 82 (2010) 116008 [arXiv:1009.5961] [INSPIRE].

[14] S. Aoki et al., Review of lattice results concerning low-energy particle physics, Eur. Phys. J. C 74 (2014) 2890 [arXiv:1310.8555] [INSPIRE]. 
[15] S. Antusch, M. Holthausen, M.A. Schmidt and M. Spinrath, Solving the Strong CP Problem with Discrete Symmetries and the Right Unitarity Triangle, Nucl. Phys. B 877 (2013) 752 [arXiv: 1307.0710] [INSPIRE].

[16] V.A. Rubakov, Grand unification and heavy axion, JETP Lett. 65 (1997) 621 [hep-ph/9703409] [INSPIRE].

[17] A. Hook, Anomalous solutions to the strong CP problem, Phys. Rev. Lett. 114 (2015) 141801 [arXiv: 1411.3325] [INSPIRE].

[18] G. Hiller and M. Schmaltz, Solving the strong CP problem with supersymmetry, Phys. Lett. B 514 (2001) 263 [hep-ph/0105254] [INSPIRE].

[19] L.M. Carpenter, M. Dine and G. Festuccia, Dynamics of the Peccei Quinn Scale, Phys. Rev. D 80 (2009) 125017 [arXiv:0906.1273] [INSPIRE].

[20] L. Bento, G.C. Branco and P.A. Parada, A minimal model with natural suppression of strong CP-violation, Phys. Lett. B 267 (1991) 95 [InSPIRE].

[21] H.E. Haber and Z. Surujon, A Group-theoretic Condition for Spontaneous CP-violation, Phys. Rev. D 86 (2012) 075007 [arXiv:1201.1730] [INSPIRE].

[22] R.F. Dashen, Some features of chiral symmetry breaking, Phys. Rev. D 3 (1971) 1879 [INSPIRE].

[23] L. Vecchi, Spontaneous CP-violation and the strong CP problem, arXiv:1412.3805 [INSPIRE].

[24] M. Kamionkowski and J. March-Russell, Planck scale physics and the Peccei-Quinn mechanism, Phys. Lett. B 282 (1992) 137 [hep-th/9202003] [INSPIRE].

[25] E. Witten, Some Properties of O(32) Superstrings, Phys. Lett. B 149 (1984) 351 [InSPIRE].

[26] M. Dine, G. Festuccia, J. Kehayias and W. Wu, Axions in the Landscape and String Theory, JHEP 01 (2011) 012 [arXiv: 1010.4803] [inSPIRE].

[27] A. Strominger and E. Witten, New Manifolds for Superstring Compactification, Commun. Math. Phys. 101 (1985) 341 [INSPIRE].

[28] K.-w. Choi, D.B. Kaplan and A.E. Nelson, Is CP a gauge symmetry?, Nucl. Phys. B 391 (1993) 515 [hep-ph/9205202] [inSPIRE].

[29] M. Dine, R.G. Leigh and D.A. MacIntire, Of CP and other gauge symmetries in string theory, Phys. Rev. Lett. 69 (1992) 2030 [hep-th/9205011] [INSPIRE].

[30] S. Kachru, R. Kallosh, A.D. Linde and S.P. Trivedi, de Sitter vacua in string theory, Phys. Rev. D 68 (2003) 046005 [hep-th/0301240] [INSPIRE].

[31] M. Dine, R.G. Leigh and A. Kagan, Supersymmetry and the Nelson-Barr mechanism, Phys. Rev. D 48 (1993) 2214 [hep-ph/9303296] [INSPIRE].

[32] S.M. Barr, Supersymmetric solutions to the strong CP problem, Phys. Rev. D 56 (1997) 1475 [hep-ph/9612396] [INSPIRE]. 